\title{
The human visual cortex responds to gene therapy-mediated recovery of retinal function
}

\author{
Manzar Ashtari, ${ }^{1}$ Laura L. Cyckowski, ${ }^{1}$ Justin F. Monroe, ${ }^{1}$ Kathleen A. Marshall, ${ }^{2}$ Daniel C. Chung,, ${ }^{2,3}$ \\ Alberto Auricchio, ,45 Francesca Simonelli, 4,5,6 Bart P. Leroy,7 Albert M. Maguire,2,3 \\ Kenneth S. Shindler, ${ }^{3}$ and Jean Bennett ${ }^{2,3}$
}

\begin{abstract}
1'Department of Radiology and ${ }^{2}$ Center for Cellular and Molecular Therapeutics, Children's Hospital of Philadelphia (CHOP), Philadelphia, Pennsylvania, USA.
3F.M. Kirby Center for Molecular Ophthalmology, Scheie Eye Institute, University of Pennsylvania School of Medicine, Philadelphia, Pennsylvania, USA.

${ }^{4}$ Telethon Institute of Genetics and Medicine (TIGEM) and 5Medical Genetics, Department of Pediatrics, "Federico II University," Naples, Italy. ${ }^{6}$ Second University of Naples, Naples, Italy. ${ }^{7}$ Department of Ophthalmology and Center for Medical Genetics, Ghent University and Ghent University Hospital, Ghent, Belgium.
\end{abstract}

\begin{abstract}
Leber congenital amaurosis (LCA) is a rare degenerative eye disease, linked to mutations in at least 14 genes. A recent gene therapy trial in patients with LCA2, who have mutations in RPE65, demonstrated that subretinal injection of an adeno-associated virus (AAV) carrying the normal cDNA of that gene (AAV2-hRPE65v2) could markedly improve vision. However, it remains unclear how the visual cortex responds to recovery of retinal function after prolonged sensory deprivation. Here, 3 of the gene therapy trial subjects, treated at ages 8, 9, and 35 years, underwent functional MRI within 2 years of unilateral injection of AAV2-hRPE65v2. All subjects showed increased cortical activation in response to high- and medium-contrast stimuli after exposure to the treated compared with the untreated eye. Furthermore, we observed a correlation between the visual field maps and the distribution of cortical activations for the treated eyes. These data suggest that despite severe and long-term visual impairment, treated LCA2 patients have intact and responsive visual pathways. In addition, these data suggest that gene therapy resulted in not only sustained and improved visual ability, but also enhanced contrast sensitivity.
\end{abstract}

\section{Introduction}

Leber congenital amaurosis (LCA) is a rare eye disease, usually inherited in an autosomal-recessive fashion. It is symptomatic at birth or within the first few months of life and affects around 1 in 81,000 people (1). LCA has been associated with at least 14 different genes $(2,3)$. The gene encoding retinal pigment epithelium-specific protein $65 \mathrm{kDa}$ (RPE65) is involved in one of the more common forms of LCA, the congenital blindness disorder LCA2 $(4,5)$. LCA2 patients are good candidates for gene transfer therapy, as the degeneration of retinal cells is delayed several years after initial symptoms. Studies in animal models of LCA2 (6-10) and, more recently, in humans (11-17) using adeno-associated virus-mediated (AAV-mediated) retinal gene delivery have demonstrated success in restoring retinal and visual function using one or more measures, including visual acuity (VA), visual fields (VFs), light sensitivity, pupillary light reflex, and/or mobility. Of all sensory systems, vision is processed by the largest brain area. Early onset of blindness can lead to structural and functional brain changes. It is not presently known whether severe impairment of the visual pathway caused by LCA2 would limit the responsiveness of neurons in the visual cortex. This question has been partially answered in a functional brain study performed in an animal model of LCA2 (18). Aguirre et al. reported cortical responses in 5 RPE65 mutant dogs after bilateral treatment, 3 untreated affected dogs, and 2 normal dogs (18). All animals underwent functional MRI (fMRI) with checkerboard pattern stimuli presented bilaterally under intense, bright light $\left(2.8 \log \mathrm{cd} \cdot \mathrm{m}^{-2}\right)$. fMRI results from

Conflict of interest: J. Bennett and A.M. Maguire are coinventors of a pending patent for a method to treat or slow the development of blindness. J. Bennett has served on scientific advisory boards for Sanofi-Aventis and Avalanche Technologies and as a consultant for GlaxoSmithKline.

Citation for this article: J Clin Invest. 2011;121(6):2160-2168. doi:10.1172/JCI57377. this study showed dramatic visual cortical response for the treated as compared to untreated dogs. Aguirre and colleagues only looked at bright light responses and also grouped the results from dogs/eyes of different ages and with different sites of injection, so that details of the retinotopic projections could not be appreciated (18). Aguirre and colleagues also performed fMRI on untreated human LCA2 subjects using stimuli of uniform light intensity varying from low to very high brightness. AMRI results showed that untreated LCA2 human subjects show cortical responses after illumination with extremely bright light and high contrast stimuli while no cortical response was observed with dim light (18). While other studies have looked at brain changes in blind-at-birth individuals or those with blindness acquired after early childhood $(19,20)$, there is limited information about brain function in human subjects following recovery of retinal function after a long period of visual deprivation.

The present study was carried out in human LCA2 patients to investigate how the cortex responds to the recovery of function after gene therapy in specific areas of the retina after prolonged visual deprivation. In contrast to the study by Aguirre et al. (18), the current study used only dim light stimuli, since it is known that young LCA2 patients are able to see and function (albeit poorly) when their environment is flooded with light (21-23). Also, to account for variability in the disease stage among study participants and to correlate fMRI results with each subject's behavioral measures, functional analyses were carried out separately for each individual patient. LCA2 subjects were injected unilaterally in their worst-seeing eye - so labeled based on both qualitative and quantitative tests as well as self reports. However, the differences in function between the eyes, based on VA, VF, light sensitivity, and imaging studies, was minimal $(16,17)$. The untreated eye served as an internal control for each subject. As an additional control, we performed $\mathrm{PMR}$ on a normally sighted individual, age and sex matched to 2 of the 3 LCA2 participants. 

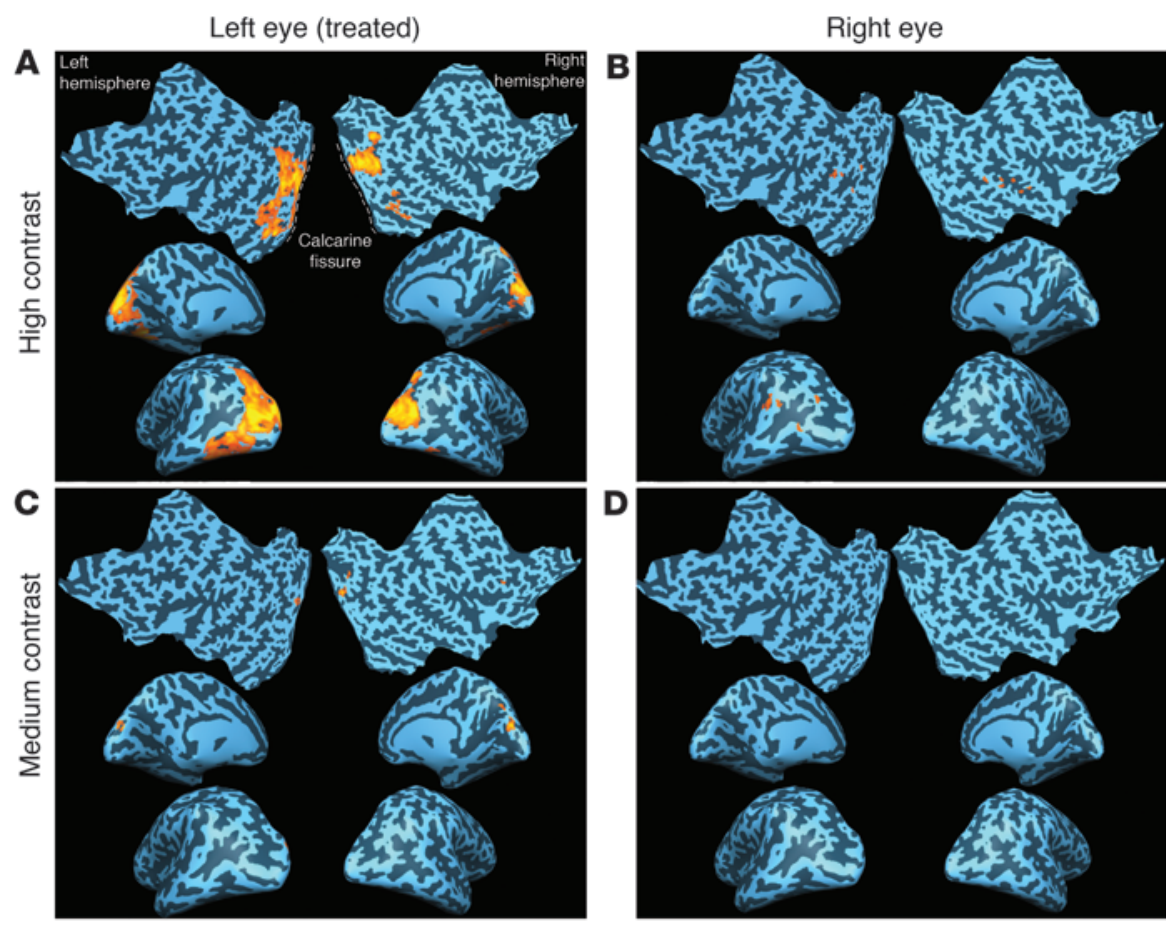

D

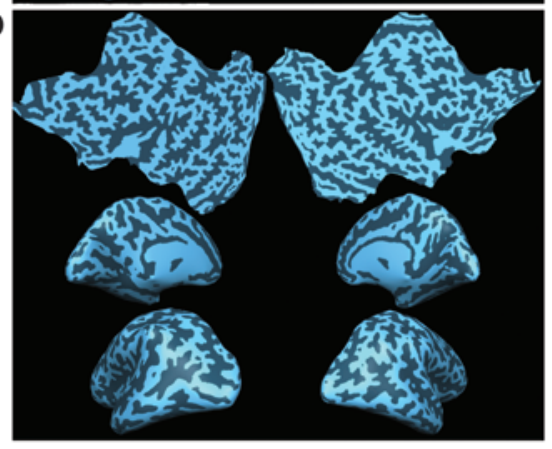

E

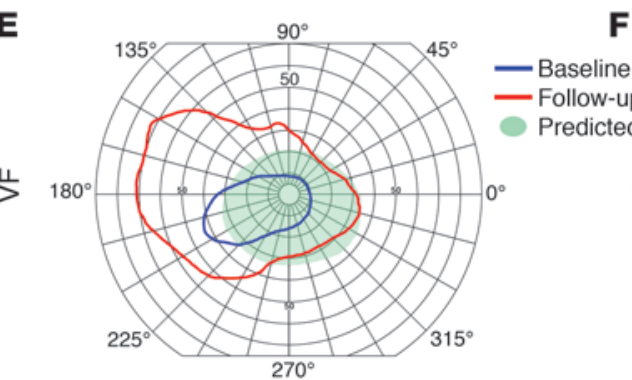

$\mathbf{F}$

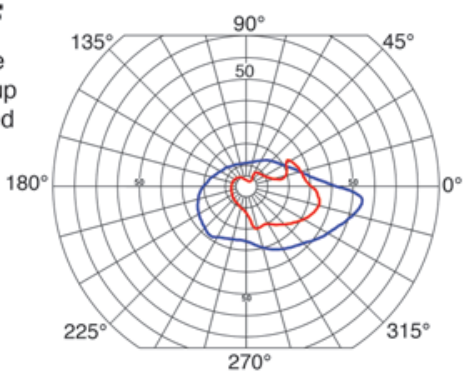

Figure 1

Results for subject CH09. (A) fMRI results for high-contrast stimulus presented to the treated left eye showed significant (fdr $<5 \%$, corrected $P<0.005$, cca $\geq 100 \mathrm{~mm}^{2}$ ) bilateral activations extending from medial to lateral aspects of the occipital poles. All fMRI results show left and right inflated (medial view) and flatmap cortical representations. (C) Medium-contrast stimulus presented to the left eye also showed significant ( $\mathrm{fdr}<5 \%$, corrected $P<0.002, \mathrm{cca} \geq 20$ $\mathrm{mm}^{2}$ ) bilateral activations. Activations for high- and medium-contrast stimuli were primarily distributed to the upper bank of the CF. (B) The high-contrast stimulus presented to the untreated right eye showed patches of activation outside the primary visual cortex at a lower (uncorrected) statistical threshold and a smaller extent threshold. (D) No activation, even at an uncorrected relaxed statistical threshold, was detected for the right eye presented with the medium-contrast stimulus. ( $E$ and $\mathbf{F}$ ) Measured VFs at baseline (before surgery) and at follow-up. (G) The predicted VF area in $\mathbf{E}$ was based on the observed subretinal injection site at the time of treatment, indicated by white arrowheads within the composite retinal fundus image. CH09's predicted VF was symmetric to the vertical meridian with a greater area below than above the horizontal meridian. Such VF distribution is predictive of cortical activation that is symmetrically distributed to both hemispheres, with a larger area of activation in the upper bank of the CF. CH09's fMRI results partially correlated with both predicted and measured VFs.

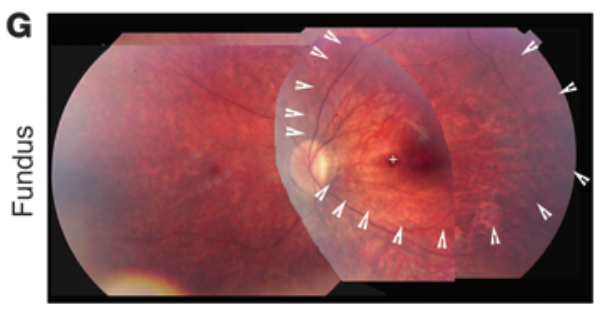

\section{Results}

\section{Clinical visual function measures}

Each subject underwent comprehensive baseline and serial postinjection retinal/visual function testing $(16,17)$ at prescribed time points (baseline; days 1-3, 14, 30, 60, 90, 180, and 270; and through day 365 for subject $\mathrm{CH} 13$, year 1.5 for $\mathrm{CH} 09$, and year 2 for $\mathrm{CH} 08$ ) after their assent/consent to enroll in the gene transfer study (16), prior to enrollment in the fMRI study. Each subject demonstrated both subjective and objective improvement in multiple parameters after administration of AAV2-hRPE65v2, including expansion of the VFs (Figures 1, 2, and 3). Improvements at day 365 (subjects $\mathrm{CH} 09$ and CH08) and day 180 (subject CH13) after injection have been previously described (16). The findings for the subjects at the time of fMRI (compared with baseline measurements) are summarized below.
Full-field sensitivity threshold testing. All subjects showed significant improvements in full-field sensitivity threshold testing in the injected eye only (16). There were greater than 10-dB differences in light sensitivity of the injected versus uninjected eyes at all postinjection time points.

Pupillometry light reflex. All subjects showed significantly improved amplitudes and velocities of constriction after illumination of the injected eye only (16). Each individual subject showed a greater than 10-fold decrease in the light intensity threshold required to generate a pupillary light reflex in their injected eye.

Ocular motility/position. There was a slight decrease in the velocity of nystagmus (but no change in amplitude) in CH08's eyes at the 1.5-year time point compared with baseline. The other 2 subjects did not have significant nystagmus at either baseline or the time of fMRI. In addition, both before and after injection, the following 

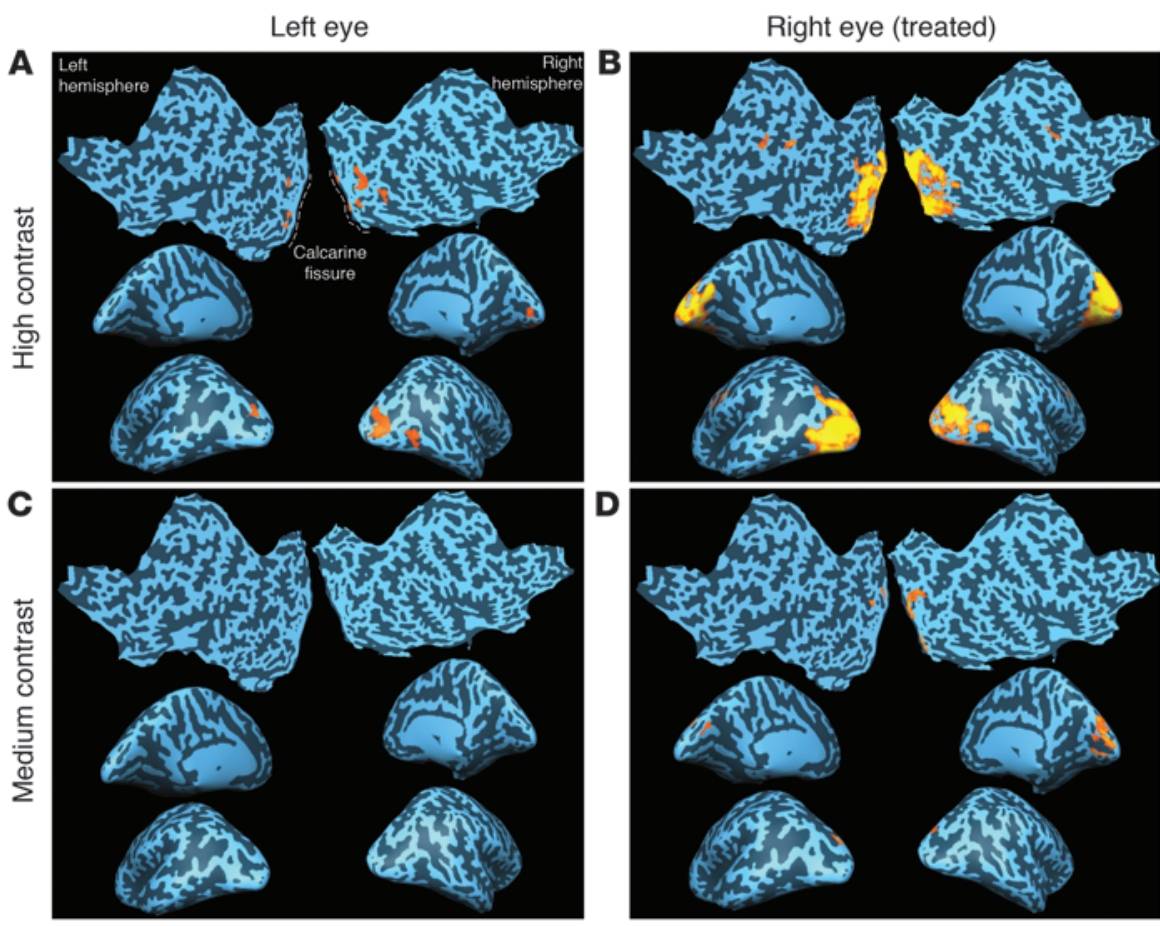

E

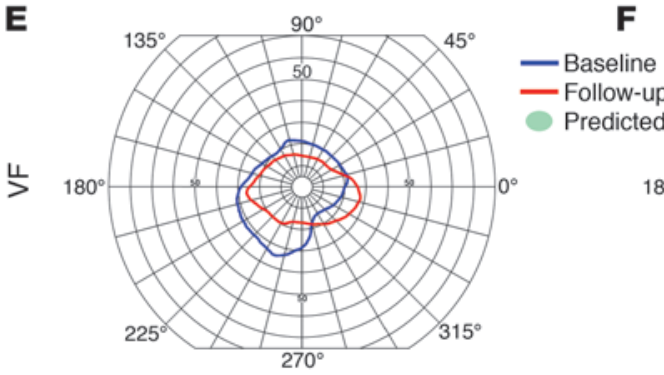

F

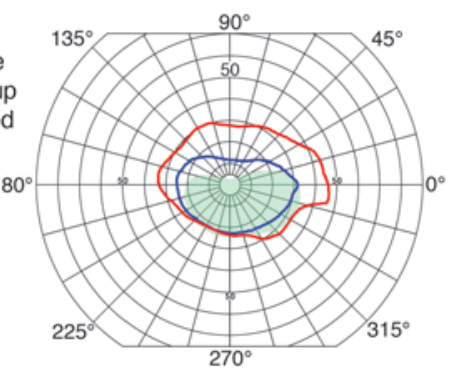

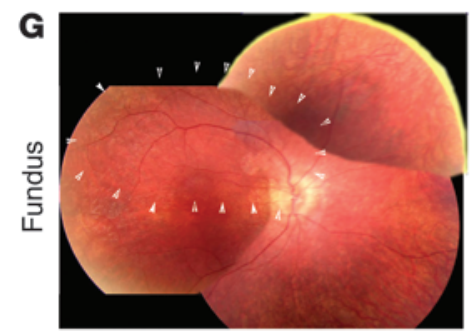

Figure 2

Results for subject $\mathrm{CH08}$. (B) fMRI results showed significant ( $\mathrm{fdr}<5 \%$, corrected $P<0.005$, cca $\geq 100 \mathrm{~mm}^{2}$ ) bilateral activations in the primary visual cortex extending from medial to lateral and posterior to anterior aspects of the occipital cortex after presentation of high-contrast stimuli to the treated right eye. (A) In contrast, the untreated left eye was nearly unresponsive to the high-contrast stimuli, with activation detectable only at a less stringent statistical threshold and much lower extent threshold (uncorrected $P<0.005$, cca $\geq 50 \mathrm{~mm}^{2}$ ). (D) Activation for medium-contrast stimuli presented to the treated eye was also bilateral, but with slightly more activation in the right hemisphere (fdr $<5 \%$, corrected $P<0.004$, cca $\geq 50 \mathrm{~mm}^{2}$ ). (C) No cortical activation was observed with the untreated eye exposed to the medium-contrast stimuli. ( $E$ and $F$ ) Measured VFs at baseline (before surgery) and at follow-up. (G) The predicted VF area in $\mathbf{E}$ was based on the observed subretinal injection site at the time of treatment, indicated by white arrowheads within the composite retinal fundus image (taken just prior to surgery). Based on the injection site located superior to the macula, the portion of the VF predicted to improve was the central inferior field. Such a VF is predictive of activation distributed bilaterally with the activation primarily located in the superior portion of the CF. CH08's fMRI results were equally distributed about the CF and correlated more with his measured VFs. However, as suggested by the predicted and measured VFs, activation was distributed roughly equally to both hemispheres. patient-specific observations were made about eye position: $\mathrm{CH} 09$, who is myopic and requires correction of 12.5 diopters, displayed a left eye exotropia when his glasses were removed; $\mathrm{CH} 13$ showed esotropia of his right eye; except for the nystagmus, there were no anomalies noted in CH08's gaze.

Mobility testing. Mobility was assessed using navigation through a standardized obstacle course (15-17). CH09 and CH08 showed improved navigation through the course and were able to navigate the course in dim light (injected eye only) (16). No significant changes were observed for $\mathrm{CH} 13$ compared to his baseline performance.

$V A$. There were no significant changes in VA in either eye in CH09 with VAs in both treated and untreated eyes of $\sim$ LogMAR $1.0(20 / 200)$ at baseline and at year 1. There was a trend of improvement (albeit not statistically significant) in CH13's VAs (both eyes), with injected eye at LogMAR 1.9 ( 20/1,589) at base- line and LogMAR $\sim 1.61(\sim 20 / 815)$ at year 1 . There was a gradual decline over time in the VA of CH08's treated eye (from LogMAR $\sim 1.31$ to $\sim 1.53$ over 2 years).

Contrast sensitivity. There were no significant changes in contrast sensitivity in any of the eyes/subjects, as assessed with the Pelli-Robson contrast sensitivity chart, prior to and after gene transfer $(16,17)$.

\section{fMRI results}

Each subject provided assent or consent to enroll in the fMRI study after subretinal injections had been administered. All fMRI analyses were performed using the general linear model (GLM) and the contrast of active blocks less the rest blocks as implemented in BrainVoyagerQX software (Methods and ref. 24). fMRI results showed substantial cortical activation in and around the visual cortex for LCA2 subjects after presentation of full-field contrast-reversing 
Left eye
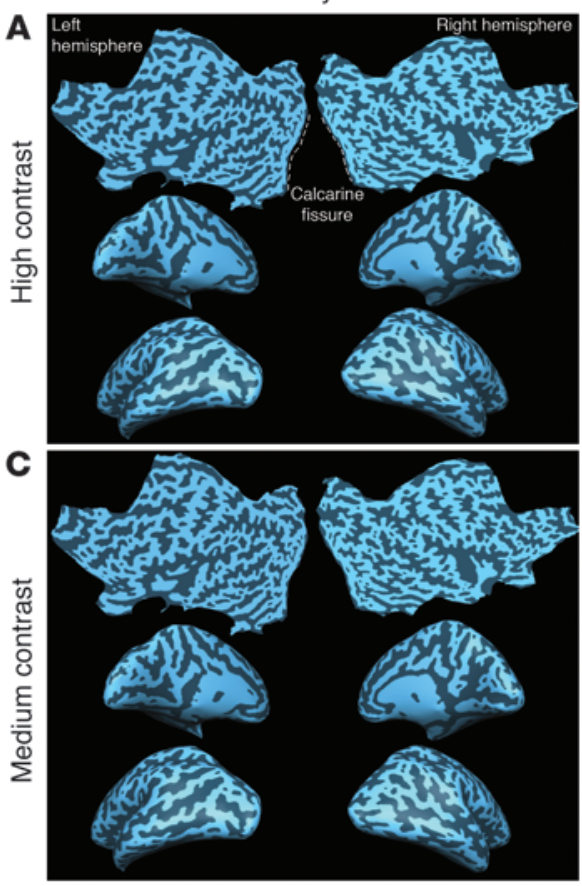

E

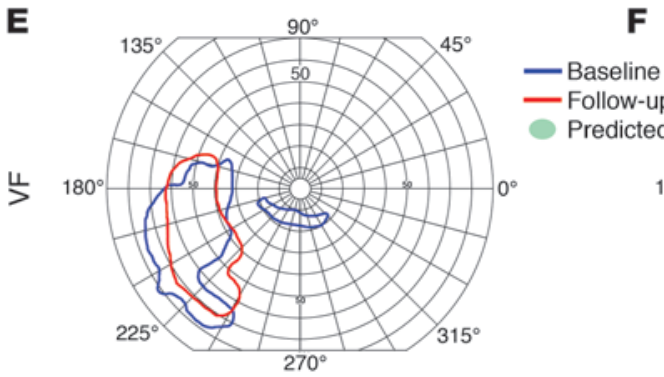

D

$\mathbf{F}$
Right eye (treated)
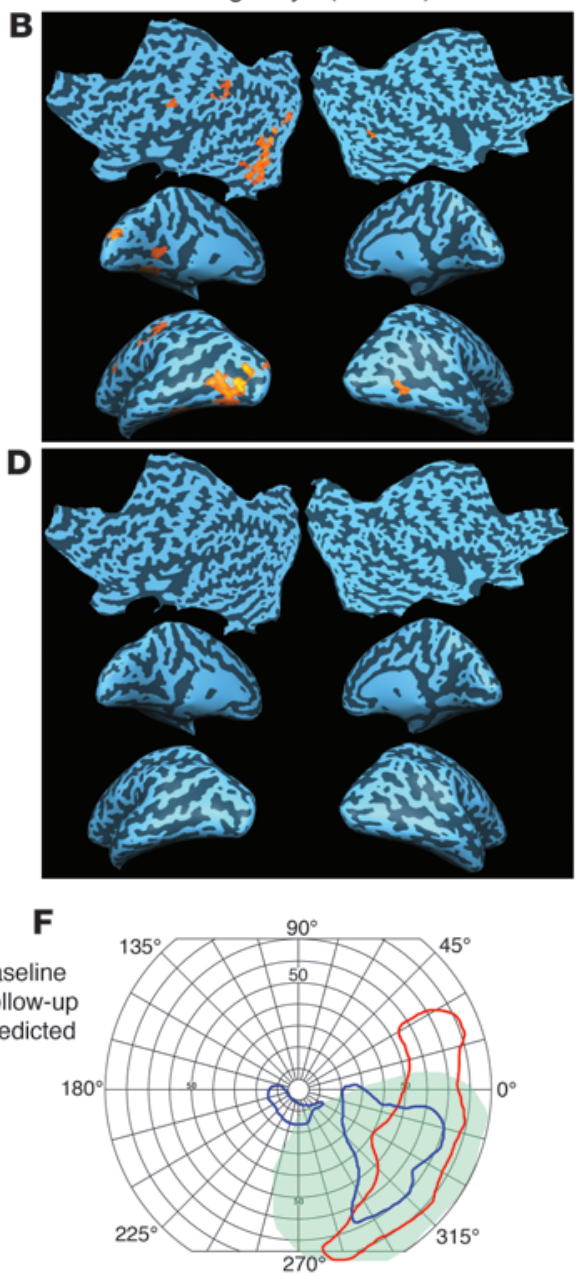

G

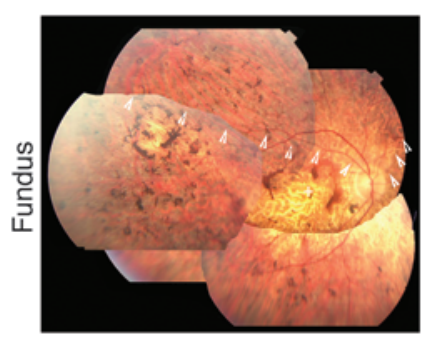

Figure 3

Results for subject $\mathrm{CH} 13$. (B) fMRI results 1 year after gene transfer for the treated right eye presented with the high-contrast stimuli showed significant (fdr $<5 \%$, corrected $P<0.003$, cca $\geq 100 \mathrm{~mm}^{2}$ ) unilateral cortical activation, primarily confined to the left lateral occipital lobe. (A) No activation was observed after exposure of the untreated left eye to the high-contrast stimuli. No activation was observed after medium-contrast stimulus was presented to the treated (D) or untreated (C) eye. (E and F) Measured and predicted VFs. The predicted VF for the treated eye showed a lateralized predicted VF in the lower right quadrant. This predicted VF was mostly confined to the right of the vertical meridian and located primarily below the horizontal meridian. Such distribution predicts cortical activation in the left upper area of the cortex, with no activation in the contralateral hemisphere. The island of activation for the treated eye occurred in the predicted hemisphere (see B), although it was located far lateral to primary visual cortex. (G) These results are consistent with the location of $\mathrm{CH} 13$ 's subretinal injection. Composite of retinal fundus images demonstrating pigmentary deposits, vascular attenuation, and "window defects" caused by degeneration particularly in the central macula. As a result, $\mathrm{CH} 13$ had a subretinal injection located more peripheral (predominantly superotemporal to the macula).
$(8 \mathrm{~Hz})$ checkerboard stimuli at high and medium contrasts to their treated eye. None of the patients showed any cortical activation for the low-contrast stimuli with their treated or untreated eye. Presentation of the same stimuli to the untreated eye of all LCA2 subjects did not result in significant cortical activations for the high- or medium-contrast stimuli.

CH09 showed highly significant (false discovery rate $[\mathrm{fdr}]<5 \%$, corrected $P<0.005$, continuous connected area [cca] $\geq 100 \mathrm{~mm}^{2}$ ), bilateral activation in response to the high-contrast stimulus for his left eye at 580 days after injection (Figure 1A). A major portion of the activation occurred in the upper bank of the calcarine fissure (CF) in both the right and left hemispheres. The medium-contrast stimulus also induced significant bilateral activation ( $\mathrm{fdr}<5 \%$, corrected $P<0.002$, cca $\geq 20 \mathrm{~mm}^{2}$ ), primarily in the upper portion of the CF (Figure 1C). As shown in Figure 1A, the main body of visual activation occurred in the lateral cortex. Although bilateral, there was a slightly greater amount of activation in the left hemisphere extending to the parietal, temporal, and ventral occipital areas. $\mathrm{CH} 09$ did not present with activation in the occipital pole, corresponding to a lack of foveal activation (Figure 1A). The subject's fMRI results for the untreated right eye with high-contrast stimulus showed patches of activation outside the primary visual cortex, at a much less stringent statistical threshold that was not corrected for multiple comparisons (uncorrected $P<0.002$, $c c a \geq 20 \mathrm{~mm}^{2}$; Figure 1B). No cortical activation was observed for the untreated eye in response to the medium-contrast stimulus, even at an uncorrected and very relaxed statistical threshold (uncorrected $P<0.01 ; \mathrm{cca} \geq 20 \mathrm{~mm}^{2}$ ).

After gene therapy, the VF of CH09 (Figure 1, E and F) was considerably extended compared with that before surgery. The predicted VF, 


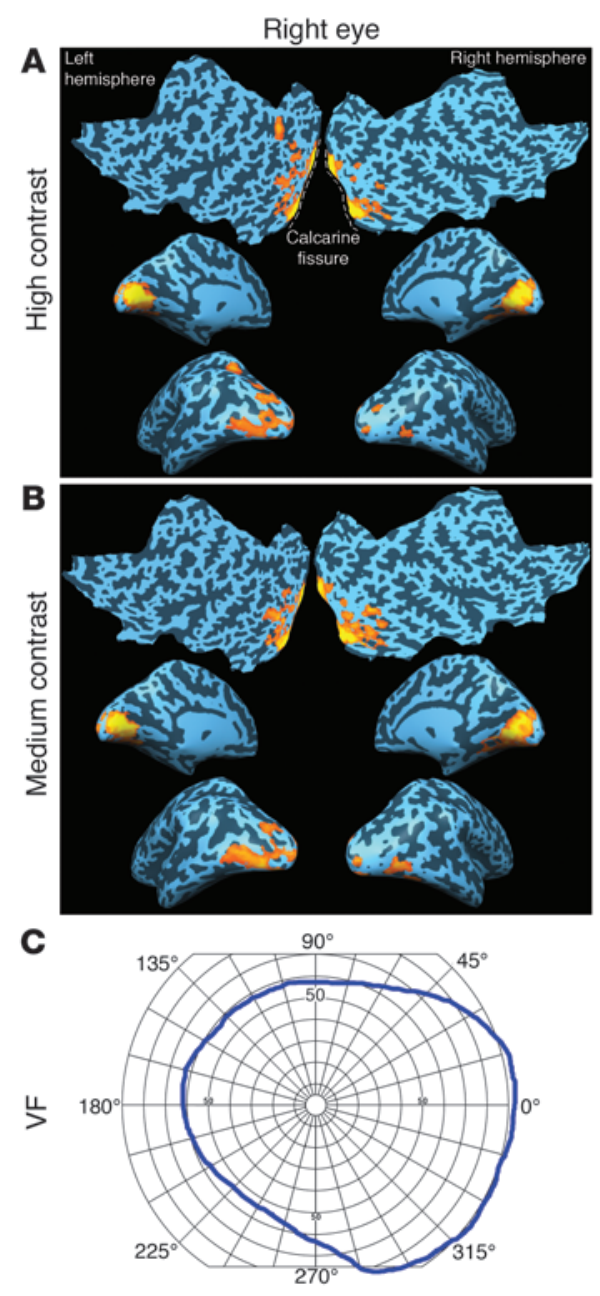

based on the observed subretinal injection site at the time of treatment (Figure 1G), was symmetric to the vertical meridian, but covered a greater area below than above the horizontal meridian (Figure 1E).

$\mathrm{CH08}$ showed highly significant bilateral activation to the highcontrast stimulus ( $\mathrm{fdr}<5 \%$, corrected $P<0.005$, cca $\geq 100 \mathrm{~mm}^{2}$ ) for his right eye (728 days after injection; Figure $2 \mathrm{~B})$. Visual activation extended from the posterior to the anterior aspects of occipital cortex on the medial, lateral, and ventral surfaces of the visual cortex. The medium-contrast stimulus for the treated eye of $\mathrm{CH} 08$ also evoked significant activation ( $\mathrm{fdr}<5 \%$, corrected $P<0.004$, cca $\geq 50$ $\mathrm{mm}^{2}$ ), which was bilateral, with slightly more activation in the right hemisphere (Figure 2D). The pattern of activation included a larger portion of activation in the superior bank of the CF. In contrast, the subject's untreated left eye was nearly unresponsive to the high-contrast stimulus, with activation detectable only at a much less stringent statistical threshold and much lower extent threshold (uncorrected $P<0.005, \mathrm{cca} \geq 50 \mathrm{~mm}^{2}$; Figure $2 \mathrm{~A}$ ). No cortical activation was observed for the untreated eye in response to the medium-contrast stimulus (Figure 2C), even at a very relaxed statistical threshold.

CH08's VF maps (Figure 2, E and F) showed considerable improvement after injection compared with baseline. Based on the area of subretinal injection, which was localized to the superior macula (Figure $2 \mathrm{G}$ ), the portion of the VF predicted to improve was the central inferior field (Figure 2E). In contrast to the sub-

\section{Figure 4}

Results for control subject NC01. Because vision tests showed normal vision for both left and right eyes, results from the right eye only are shown. fMRI results showed significant ( $\mathrm{fdr}<5 \%$, corrected $P<0.003, \mathrm{cca} \geq 100 \mathrm{~mm}^{2}$ ) bilateral activations in the primary visual cortex extending from posterior to anterior and medial to some lateral aspects of the occipital cortex after presentation of high-contrast (A) and medium-contrast (B) stimuli to his right eye. Medial cortex activation was distributed symmetrically to the upper and lower banks of the CF in both the right and left hemispheres. Similar to the LCA2 participants, and as predicted based on the scotopic light stimulus and/or the size of squares used in the checkerboard stimuli, NC01 did not show any activation in the occipital pole, where foveal activation would otherwise be represented. (C) VF map, which shows symmetrical distribution with respect to the horizontal meridian but covers a greater area to the right of the vertical meridian. Such VF is predictive of bilateral $\mathrm{fMRI}$ activations that are equally spread to the superior and inferior aspects of CF. In agreement with his VF, activation was distributed equally to superior and inferior aspects of CF for both hemispheres. Also, as predicted by his VF, activations were bilateral with slightly more activation in the left lateral visual cortex.

ject's treated eye, the follow-up VF of the untreated eye was smaller than that at baseline (Figure 2F).

CH13's fMRI results for the high-contrast stimulus showed significant (fdr $<5 \%$, corrected $P<0.003$, cca $\geq 100 \mathrm{~mm}^{2}$ ) cortical activation in the left lateral and ventral occipital lobe (370 days after injection; Figure 3B). The clusters of activation for $\mathrm{CH} 13$ were primarily anterior in location, with almost no activation in the posterior aspects of the occipital cortex. The subject did not demonstrate any activation for the medium-contrast stimulus presented to the treated right eye (Figure 2D). In contrast to his treated eye, there was no cortical activation, even at an uncorrected and very relaxed statistical threshold, for the untreated left eye for either high- or medium-contrast stimulus (uncorrected $P<0.01$; cca $\geq 50 \mathrm{~mm}^{2}$; Figure 3, A and C).

Comparison of pre- and postinjection VFs in $\mathrm{CH} 13$ showed progressive VF loss in the untreated eye (Figure $3 \mathrm{E}$ ). The VF in the treated eye expanded considerably after subretinal injection (Figure 3F). Because of the advanced disease in CH13, AAV2-hRPE65v2 was delivered supratemporally to the macula of his right eye, an area with better retinal quality (Figure $3 \mathrm{G}$ ). The VF predicted based on the injection site was localized to the right hemifield and was primarily inferior (Figure 3F).

We also performed vision tests for a 10 -year-old normal control subject, NC01 (age and sex matched to LCA2 patients CH08 and CH09), which showed normal vision for both right and left eyes (Figure 4 and data not shown). The subject demonstrated significant bilateral activation for the high-contrast stimulus for his right eye (fdr $<5 \%$, corrected $P<0.003, \mathrm{cca} \geq 100 \mathrm{~mm}^{2}$; Figure 4A). NC01's visual activation extended from the posterior to the anterior aspects of the occipital cortex, primarily on the medial and partially on the lateral and ventral surfaces of the visual cortex. Activation was distributed symmetrically to the upper and lower banks of the CF in both right and left hemispheres. The mediumcontrast stimulus also induced significant bilateral activation (fdr $<5 \%$, corrected $P<0.002, \mathrm{cca} \geq 200 \mathrm{~mm}^{2}$; Figure 4B). Similar to the high-contrast stimulus, fMRI activation for the medium-contrast stimulus was distributed from the posterior to the anterior aspects of the occipital cortex. Similar to the LCA2 participants, NC01 did not present with any activation in the occipital pole 

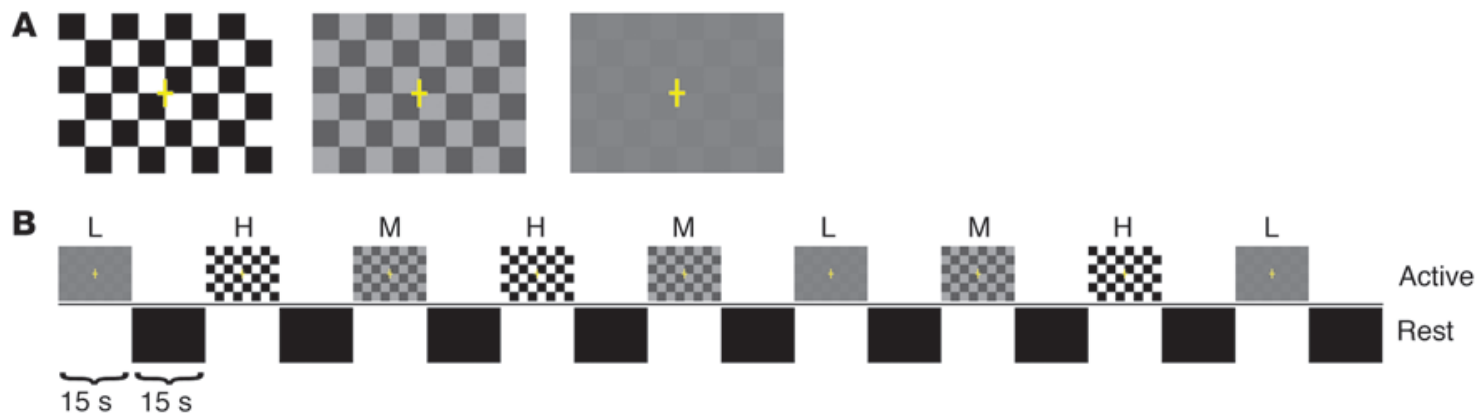

\section{Figure 5}

fMRI stimuli and design. (A) Checkerboard stimuli with a constant light intensity of 5 lux, at 3 levels of contrast (high, $100 \%$; medium, $34 \%$; low, $10 \%$ ), were presented in a boxcar block design. (B) The checkerboard paradigm consisted of 15 -second active blocks of contrast-reversing $(8 \mathrm{~Hz})$ checkerboards interleaved with 15-second presentation of a blank (black) screen as control blocks (rest period). 3 blocks of each contrast were interspersed randomly and interleaved with 9 rest blocks. Subjects were asked to press a button when they detected a checkerboard pattern. L, low contrast; $\mathrm{M}$, medium contrast; $\mathrm{H}$, high contrast.

(foveal activation). NC01 presented with ventral and temporal activation for both high and medium contrasts, with a slightly greater distribution in the left hemisphere. The subject's VF showed a symmetrical distribution with respect to the horizontal meridian covering a greater portion of the right VF (Figure 4C). Similar cortical activation was observed in 2 additional normal-sighted controls, not age and sex matched (data not shown).

\section{Discussion}

To our knowledge, it was not previously known whether severe impairment of the visual pathway caused by congenital or earlyonset inherited retinal degeneration limits the responsiveness of neurons in the visual cortex that normally process vision. Here we showed definitively, through a combination of psychophysical testing and $\mathrm{AMRI}$ measurements, that gene augmentation therapy in humans with LCA2 rendered the retina (and visual cortex) far more sensitive to dimmer light and lower-contrast stimuli.

The site of injection for each patient varied according to the progression of the disease and the area of the retina in which there was evidence of sufficient (albeit unhealthy) retinal cells $(16,17)$. Since results were analyzed individually for each eye/patient (and not grouped, as is done in most other studies), it was possible to correlate the retinal injection site with the area of cortical activation. The fMRI experiment used dim light stimuli that had been imperceptible by the subjects prior to intervention (and remained imperceptible to the untreated eye).

As predicted, based on the retinal function tests, cortical activation was generally observed in regions corresponding to the area of retina that had been exposed to AAV2-hRPE65v2 by injection and immediate postinjection positioning. For $\mathrm{CH} 09$, $\mathrm{AMRI}$ results from his treated eye showed bilateral activations for high- and mediumcontrast stimuli. Based on the retinal injection site in the superior macula, the subject had a predicted VF that was symmetrical with respect to the vertical meridian and covered a greater area below than above the horizontal meridian. Such VF distribution is predictive of bilateral cortical activations with activations more distributed to the superior portion of the CF than the inferior portion for both hemispheres. CH09's pattern of activations partially correlated with the predicted VF: activations were more distributed to the superior portion of the CF and were bilateral. However, interestingly, more activation was observed in the left hemisphere. Although the reason for this asymmetric distribution is not known, it may suggest some level of brain plasticity, or it may be due to the degree of retinal cell degenerations distributed along his retina. For example, if retinal cells were more viable and healthier in the temporal as opposed to nasal retina, it would produce greater ipsilateral cortical activation as opposed to contralateral activation.

CH08 presented with highly significant bilateral activations for the high- and medium-contrast stimuli presented to his treated eye. Compared with that of CH09, CH08's activation was symmetrically distributed and more significant. Observed differences in activation between $\mathrm{CHO}$ and $\mathrm{CH} 09$ may be attributed to the fact that $\mathrm{CH} 08$ had a thicker retina, identified at the time of surgery, or may be due to longer recovery after treatment for $\mathrm{CH} 08$ (2 years) compared with CH09 (1.5 years). Like CH09, CH08's injection was delivered centrally (superior macula), and the VF predicted by the injection site extended inferiorly to the horizontal meridian and stretched to the lower-right quadrant. As suggested by the predicted field, $\mathrm{CH} 08$ presented with bilateral activation; however, the distribution of the visual activation correlated even better with the measured Goldmann VF, with activation extended from the posterior to the anterior visual cortex and distributed equally superior and inferior to the CF for both hemispheres, with slightly more activation in the left hemisphere.

Given the lack of viable cells in the macula of subject CH13 (Figure $3 \mathrm{G}$ ), the patient had a subretinal injection located more peripheral than those in $\mathrm{CH} 09$ and $\mathrm{CH} 08$. For $\mathrm{CH} 13$, cortical activation was confined to the left hemisphere, corresponding to projections from the nasal retina in the treated eye. However, although activation was located only in the left occipital cortex, it was more lateral and basal than expected. This may represent activation of association cortex processing inputs to primary visual cortex that were below the level of detection, or increased attention to the button press during the task $(25,26)$. Alternatively, it could indicate plasticity of cortical neuronal connectivity due to the disease/treatment. In any case, the results from this subject with severe retinal/macular degenerative changes provide hope for gene therapy-mediated restoration of at least some visual function (i.e., VFs, light sensitivity) in other patients with end-stage LCA or retinitis pigmentosa.

Preservation of expected retinotopy has been identified in another patient who had partial vision restored in adulthood after having been blinded in early childhood $(19,20)$. Functional reorganiza- 
tion has been reported after long-term deprivation from stroke, retinitis pigmentosa, macular degeneration (Stargardt disease or cone dystrophy), or traumatic injury to the eye $(19,20,27-30)$. Although results are suggestive of some degree of brain plasticity around the occipital lobe within the higher-order visual cortex, our results for the most part showed preserved visual cortex in LCA2 patients. It may be that retinotopic representation is less altered in LCA2 patients until late in life, since these individuals have (limited) vision early in life.

fMRI results for CH13's treated eye showed significant activation for only the high-contrast stimuli. In contrast, $\mathrm{CH} 08$ and $\mathrm{CH} 09$ demonstrated activation in response to both high- and mediumcontrast stimuli presented to the treated eye. The larger response to high and medium-contrast stimuli in younger patients may be due to the fact that degenerative retinal changes had not progressed as far as they had in $\mathrm{CH} 13$. Moreover, the younger patients were injected in or around the macula, which may have enhanced their contrast sensitivity. Indeed, the macula is known to have a higher concentration of cone versus rod photoreceptors, a denser array of photoreceptors than the peripheral retina, and postreceptoral organization that favors higher spatial acuity $(8,31)$.

Our fMRI results showed no foveal activation in any of the patients with high- or medium-contrast stimuli. This may be due to lower spatial resolution used for the stimuli $(25,32)$ or to the scotopic light intensity of the fMRI stimuli. These explanations are supported by results from a normally sighted age- and sex-matched control, in which there was a similar lack of foveal activation. Interestingly, NC01's fMRI results showed activation similar in pattern and magnitude for the high- and medium-contrast stimuli, whereas the LCA2 subjects showed greater responses for the high-contrast stimuli. This suggests that the treated eye in LCA2 patients, even in young subjects, is still not as sensitive to lower-contrast stimuli as in normal individuals - at least within the time period evaluated. The presence of nystagmus in each patient may have influenced the fMRI results by dictating the amount of time the eye can fixate on a particular object. Alternatively, amblyopia, which is known to affect the degree of cortical activations (26), may have played a role in the level of improvement in the LCA patients. Interestingly, psychophysical testing of contrast sensitivity before and after treatment had not revealed changes in any of the subjects $(16,17)$. PMRI may therefore be more sensitive to changes in contrast sensitivity than psychophysical testing in these patients with poor VA.

In summary, our results show that in humans with LCA caused by $R P E 65$ mutations, the visual cortex can be made responsive to visual input, even after prolonged (up to 35 years) visual deprivation. To our knowledge, this is the first demonstration of stable retinotopic improvement (lasting at least 2 years) in humans after retinal gene therapy, reflected by response of the visual cortex. Our study showed not only that the gene therapy-treated retinal cells were more sensitive to dim light than were untreated cells, as reflected by visual cortex activation, but that treated patients also detected lower-contrast objects better with their treated eyes. Future studies will involve fMRI before and after gene therapy in both eyes; we expect such a comparison to provide further evidence supporting the effectiveness of gene therapy for LCA2. Finally, the fMRI finding that the visual cortex can be resuscitated after chronic visual deprivation in a rare disease has broader implications. Restoration of cortical function in LCA2 through gene augmentation therapy provides promise for the outcome of gene therapy for other, more common, retinal degenerative diseases with early or late onset.

\section{Methods}

Subjects, vector, and surgical delivery. All subjects included in this study had LCA2 and were enrolled in a phase I-II open-label dose-escalation study of the safety and efficacy of a single subretinal delivery of AAV2-hRPE65v2 (www.clinicaltrials.gov identifier NCT00516477; refs. 16, 17). The participants were 2 affected children, $\mathrm{CH} 09$ and $\mathrm{CH} 08$ (8 and 9 years of age, respectively, at the time of surgery), 1 adult, $\mathrm{CH} 13$ (35 years of age at the time of surgery), and 1 unaffected normal control child, NC01 (10 years of age). The specific RPE65 mutations in $\mathrm{CH} 09, \mathrm{CH} 08$, and $\mathrm{CH} 13$ were R124X/Lys297del1aggA, F530fs/F530fs, and IVS1 + 5g>a/IVS1 + 5g>a, respectively. $\mathrm{CH} 09$ and $\mathrm{CH} 08$ had received $4.8 \times 10^{10}$ vector genomes of AAV2-hRPE65v2 in a volume of $150 \mu \mathrm{l}$, and $\mathrm{CH} 13$ received $1.5 \times 10^{11}$ vector genomes in a volume of $300 \mu \mathrm{l}(16,17)$. The injections were performed in the more impaired eye of each individual: the left eye of $\mathrm{CH} 09$ and the right eyes of $\mathrm{CH} 08$ and $\mathrm{CH} 13(16,17)$.

The locations of retinal detachments created by the subretinal injections were recorded with an intraoperative video. Landmarks were identified, and the borders of the detachment were later transferred to a composite of retinal fundus images acquired at baseline. These borders are likely an approximation, however, because immediately after injection, each subject was positioned for optimal exposure to AAV as well as the reattachment of the retina. The retinas had all reattached by the first postoperative exam (15 hours later; refs. 16, 17). Each subject underwent comprehensive baseline and serial postinjection retinal/visual function testing $(16,17)$ at prescribed time points (see Results) prior to enrollment in the fMRI study. Goldmann VF exams (using the V4e target, the only target the subjects could see at baseline) were carried out at baseline and 1 day before fMRI testing. fMRI studies were carried out at long-term postinjection time points ( $\mathrm{CH} 13,1.0$ years; $\mathrm{CH09}, 1.5$ years; $\mathrm{CH} 08$, 2 years) after subjects provided assent with parental permission ( $\mathrm{CH} 09$ and $\mathrm{CH} 08$ ) or informed consent (CH13). Normally sighted controls were recruited to undergo vision testing and fMRI after parental permission and child assent. Results from control subject $\mathrm{NCO} 1$ (matched in age and sex to $\mathrm{CHO}$ and $\mathrm{CH} 09$ ) are shown. This study was performed according to procedures approved by the Institutional Review Board of CHOP.

Image acquisition. All $\mathrm{fMRI}$ data and $3 \mathrm{D}$ high-resolution structural brain images were acquired on a Siemens 3T Verio system at CHOP using a 32-channel head coil. Functional data were acquired using the blood oxygenation level-dependent technique, acquiring 46 slices with $3 \mathrm{~mm}$ isotropic resolution (matrix, $64 \times 64$; TR/TE, $3,000 / 30 \mathrm{~ms}$ ) with a total acquisition time of 4 minutes, 33 seconds. 5 brain volumes were acquired during both active and control blocks for a total of 90 functional volumes. 3 additional volumes were acquired at the beginning of the fMRI experiment to reach $T_{1}$ equilibrium; these were not used in image analysis. A transistor-transistor logic (TTL) pulse was used to automatically start the stimuli in sync with the start of $\mathrm{AMRI}$ acquisition.

In addition to $\mathrm{PMRI}$, isotropic $3 \mathrm{D}$ structural high-resolution $(1 \times 1 \times 1$ $\mathrm{mm}) \mathrm{T}_{1}$-weighted images of the whole brain were obtained for accurate anatomical representation of visual activation and generation of inflated hemispheres. Resonance Technology VisuaStim goggles, featuring a digital display of 500,000 pixels per 0.25 square inch and a $30^{\circ}$ horizontal field of view, was used to present the fMRI stimuli unilaterally to each eye. An MRI-compatible response device (a button that the subject pushed when recognizing the stimulus) was used to record subject responses. All visual paradigms were programmed in E-Prime (Psychology Software Tools Inc.), and all button presses were captured via a log file created by E-Prime. Subjects' button-press responses were compared with their fMRI responses to determine whether there was a correlation between the subject's report of seeing the object and activation of response in the visual cortex. 
fMRI visual paradigms. Checkerboard patterns with a constant light intensity of 5 lux were used in a block design fMRI paradigm, with 3 levels of contrast: high, 100\%; medium, 34\%; low, 10\% (Figure 5A). This paradigm consisted of 15 -second active blocks of contrast-reversing $(8-\mathrm{Hz})$ checkerboards interleaved with 15 seconds of blank (black) screens as control blocks. Subjects were asked to fixate on the plus sign in the center of the pattern, or, if they could not see this, were asked to look straight ahead. Additionally, subjects were asked to press a button as soon as they could detect checkerboard patterns. Stimuli were presented to 1 eye only; thus, each subject completed the task twice, once for each eye. Figure 5B shows a schematic diagram of the boxcar design that consisted of 3 blocks for each contrast, for a total of 9 active blocks interspersed randomly and interleaved with 9 rest blocks.

fMRI processing. All functional data were processed using BrainVoyagerQX (Brain Innovations; ref. 24). Preprocessing of data included slice scan time correction, 3D motion correction, spatial smoothing, and temporal filtering. Sinc interpolation was used for scan time correction to ensure that all voxels in the volume represented the signal simultaneously. A high-pass temporal filter of 2 cycles per run was applied to remove signal drift. Spatial smoothing was performed using a 3-mm full-width at half-maximum (FWHM) Gaussian filter. To rule out excessive motion, all functional data sets were additionally processed using the motion correction algorithm implemented in BrainVoyagerQX, which calculates head translation (in millimeters) and rotation (in degree) for each volume in relation to the first volume. None of the subjects showed excessive motion ( $\pm 1 \mathrm{~mm}$ or greater) during the scan.

Subsequent to preprocessing, functional data were coregistered to the individual's high-resolution $3 \mathrm{D}$-structural $\mathrm{T}_{1}$-weighted images using the registration routines in BrainVoyagerQX (24). fMRI results were presented as the medial and lateral surfaces of the inflated cortex and flatmap representations of each hemisphere. Superimpositions of AMRI activations on the inflated and flattened cortex provided additional information with improved visualization for cortical activity. Inflated cortices were created using an automatic segmentation algorithm implemented in BrainVoyagerQX 2.2. Prior to inflation and flattening, the high-resolution 3D images were corrected for intensity inhomogeneity using the Vaughan et al. correction algorithm (33). Next, the gray matter/white matter border was segmented using a region-growing method and analysis of intensity histograms. Morphological operations were used to smooth the borders of the segmented data and to separate the left from the right hemisphere. If necessary, manual corrections were made to obtain correct segmentation results. Each segmented hemisphere was finally submitted to a "bridge removal" algorithm, which ensures the creation of topologically correct mesh representations (34). The borders of the 2 resulting segmented subvolumes were tessellated to produce a surface reconstruction of each hemisphere. Finally, using a fully automatic 3D morphing algorithm (35), the resulting meshes were transformed into inflated and flattened cortex representations. Morphed surfaces were linked to the folded reference meshes so that functional data are shown accurately and precisely at the correct location on the inflated and flattened representations. This link was also used to keep geometric distortions to a minimum during inflation and flattening through inclusion of a morphing force that keeps the distances between vertices and the area of each triangle of the morphed surface as close as possible to the respective values of the reference mesh.

Statistics. All functional data were analyzed in BrainVoyagerQX using the GLM, which is mathematically identical to a multiple regression analysis and calculates a univariate $t$ statistic for each voxel in the dataset. Each condition (high, medium, and low contrast) was analyzed by specifying a design matrix defined as active versus rest conditions followed by application of the hemodynamic response function. However, using the GLM approach in this manner leads to a multiple-comparison problem stemming from the thousands of $t$ tests run (1 per voxel). To account for this problem, we used the fdr approach (36) as implemented in BrainVoyagerQX. Given a desired fdr, the fdr algorithm calculates a single-voxel threshold, which ensures that the voxels beyond that threshold contain no more than the specified proportion of false positives. A conservative fdr threshold of $5 \%$ $(q=0.05)$ was chosen for all analyses. The corresponding $P$ values were reported for each subject/condition, automatically calculated by BrainVoyagerQX and based on the division of the probabilities of single tests by the number of independent tests. Additionally, cluster thresholds (i.e., ccas) were defined to further control for multiple comparisons. We chose relaxed thresholds of $20-100 \mathrm{~mm}^{2}$, which allowed for the detection of relatively small but still significant areas of activity in the case of untreated eyes, while activity in the treated eye greatly exceeded this threshold (e.g., $>1,000 \mathrm{cca}$ ). Correlations between the activations in the visual cortex and the VF maps (predicted and measured) were performed qualitatively and based on published retinotopic mapping (37-39).

\section{Acknowledgments}

This study was supported by grant R21EY020662 from the National Eye Institute. This study was also funded in part by the Center for Cellular and Molecular Therapeutics (CCMT) at CHOP, the Foundation Fighting Blindness-sponsored CHOP-PENN Pediatric Center for Retinal Degenerations, Clinical Translational Science Award NIH/NCRR UL1-RR-024134, 1R01EY019014-01A2, Research to Prevent Blindness, the Paul and Evanina Mackall Foundation Trust at Scheie Eye Institute, anonymous donors, the Italian Telethon Foundation, and the F.M. Kirby Foundation. B.P. Leroy is a Senior Clinical Investigator of the Research Foundation-Flanders (Belgium) (FWO) and is further supported by FWO grant OZP 3G004306. We thank Jamie Rundio, Peter Lam, Rachel Golembski, and Xiaosong Zhu for their expert technical assistance.

Received for publication February 1, 2011, and accepted in revised form March 29, 2011.

Address correspondence to: Manzar Ashtari, Diffusion Tensor Image Analyses and Brain Morphometry Center, Children's Hospital of Philadelphia, Department of Radiology, 34th and Civic Center Blvd., 2nd floor - Wood Bld. - Room 2115, Philadelphia, Pennsylvania 19104, USA. Phone: 267.426.5690; Fax: 215.590.1345; E-mail: ashtari@email.chop.edu.
1. Stone EM. Leber congenital amaurosis - a model for efficient genetic testing of heterogeneous disorders: LXIV Edward Jackson Memorial Lecture. AmJ Ophthalmol. 2007;144(6):791-811.

2. den Hollander AI, Roepman R, Koenekoop RK, Cremers FP. Leber congenital amaurosis: genes, proteins and disease mechanisms. Prog Retin Eye Res. 2008;27(4):391-419.

3. den Hollander AI, Black A, Bennett J, Cremers FP. Lighting a candle in the dark: advances in genetics and gene therapy of recessive retinal dystrophies. J Clin Invest. 2010;120(9):3042-3053.

4. Marlhens F, et al. Mutations in RPE65 cause Leber's congenital amaurosis. Nature Genetics. 1997; 17(2):139-141.

5. Gu SM, et al. Mutations in RPE65 cause autosomal recessive childhood-onset severe retinal dystrophy. Nat Genet. 1997;17(2):194-197.

6. Acland GM, et al. Long-term restoration of rod and cone vision by single dose $\mathrm{AAV}$-mediated gene transfer to the retina in a canine model of childhood blindness. Mol Ther. 2005;12(6):1072-1082.

7. Acland GM, et al. Gene therapy restores vision in a canine model of childhood blindness. Nat Genet. 2001;28(1):92-95

8. Dejneka N, et al. Fetal virus-mediated delivery of the human RPE65 gene rescues vision in a murine model of congenital retinal blindness. Mol Ther. 2004; 9(2):182-188.

9. Bennicelli J, et al. Reversal of blindness in animal 
models of leber congenital amaurosis using optimized AAV2-mediated gene transfer. Mol Ther. 2008; 16(3):458-465.

10. Narfstrom K, Ehinger B, Bruun A. Immunnohistochemical studies of cone photoreceptors and cells of the inner retina in feline rod-cone degeneration. Vet Ophth. 2001;4(2):141-145.

11. Banin E, et al. Molecular anthropology meets genetic medicine to treat blindness in the north african jewish population: human gene therapy initiated in Israel. Hum Gene Ther. 2010;21(12):1749-1757.

12. Hauswirth W, et al. Treatment of leber congenital amaurosis due to RPE65 mutations by ocular subretinal injection of adeno-associated virus gene vector: short-term results of a phase I trial. Hum Gene Ther. 2008;19(10):979-990.

13. Cideciyan AV, et al. Human gene therapy for RPE65 isomerase deficiency activates the retinoid cycle of vision but with slow rod kinetics. Proc Natl Acad Sci US A. 2008;105(39):15112-15117.

14. Bainbridge JW, et al. Effect of gene therapy on visual function in Leber's congenital amaurosis. NEngl J Med. 2008;358(21):2231-2239.

15. Simonelli F, et al. Gene therapy for Leber's congenital amaurosis is safe and effective through 1.5 years after vector administration. Mol Ther. 2010; 18(3):643-650.

16. Maguire AM, et al. Age-dependent effects of RPE65 gene therapy for Leber's congenital amaurosis: a phase 1 dose-escalation trial. Lancet. 2009; 374(9701):1597-1605.

17. Maguire AM, et al. Safety and efficacy of gene transfer for Leber's congenital amaurosis. N Engl J Med. 2008;358(21):2240-2248.

18. Aguirre GK, et al. Canine and human visual cortex intact and responsive despite early retinal blindness from RPE65 mutation. PLoS Med. 2007;4(6):e230.
19. Fine I, et al. Long-term deprivation affects visual perception and cortex. Nat Neurosci. 2003;6(9):915-916.

20. Levin N, Dumoulin SO, Winawer J, Dougherty RF, Wandell BA. Cortical maps and white matter tracts following long period of visual deprivation and retinal image restoration. Neuron. 2010;65(1):21-31.

21. Galvin JA, Fishman GA, Stone EM, Koenekoop RK. Evaluation of genotype-phenotype associations in leber congenital amaurosis. Retina. 2005; 25(7):919-929.

22. Lorenz B, et al. Early-onset severe rod-cone dystrophy in young children with RPE65 mutations. Invest Ophthalmol Vis Sci. 2000;41(9):2735-2742.

23. Jacobson SG, et al. Defining the residual vision in leber congenital amaurosis caused by RPE65 mutations. Invest Ophthalmol Vis Sci. 2009;50(5):2368-2375.

24. Goebel R, Esposito F, Formisano E. Analysis of functional image analysis contest (FIAC) data with brainvoyager QX: From single-subject to cortically aligned group general linear model analysis and self-organizing group independent component analysis. Hum Brain Mapp. 2006;27(5):392-401.

25. Somers DC, Dale AM, Seiffert AE, Tootell RB. Functional MRI reveals spatially specific attentional modulation in human primary visual cortex. Proc Natl Acad Sci US A. 1999;96(4):1663-1668.

26. Masuda Y, et al. Task-dependent V1 responses in human retinitis pigmentosa. Invest Ophthalmol Vis Sci. 2010;51(10):5356-5364

27. Baker CI, Dilks DD, Peli E, Kanwisher N. Reorganization of visual processing in macular degeneration: replication and clues about the role of foveal loss. Vision Res. 2008;48(18):1910-1919.

28. Baker CI, Peli E, Knouf N, Kanwisher NG. Reorganization of visual processing in macular degeneration. J Neurosci. 2005;25(3):614-618.

29. Masuda Y, Dumoulin SO, Nakadomari S, Wandell
BA. V1 projection zone signals in human macular degeneration depend on task, not stimulus. Cereb Cortex. 2008;18(11):2483-2493.

30. Baseler HA, Brewer AA, Sharpe LT, Morland AB, Jagle H, Wandell BA. Reorganization of human cortical maps caused by inherited photoreceptor abnormalities. Nat Neurosci. 2002;5(4):364-370.

31. Agarwal S, Agarwal A, Apple D, Burrato L, Alió JL. Textbook of Ophthalmology. Vol. 1. Philadelphia, Pennsylvania, USA: Lippincott Williams and Wilkins; 2002.

32. Tootell RBH, Hadjikhani NK, Mendola JD, Marrett $\mathrm{S}$, Dale AM. From retinotopy to recognition: $\mathrm{AMRI}$ in human visual cortex. Trends in Cognitive Sciences. 1998; 2(5):174-183.

33. Vaughan JT, et al. 7T vs. 4T: RF power, homogeneity, and signal-to-noise comparison in head images. Magn Reson Med. 2001;46(1):24-30.

34. Kriegskorte N, Goebel R. An efficient algorithm for topologically correct segmentation of the cortical sheet in anatomical MR volumes Neuroimage. 2001; 14(2):329-346.

35. Goebel R. A fast automated method for flattening cortical surfaces. Neuroimage. 2000;11:S680.

36. Genovese CR, Lazar NA, Nichols T. Thresholding of statistical maps in functional neuroimaging using the false discovery rate. Neuroimage. 2002; 15(4):870-878.

37. Warnking J, et al. fMRI retinotopic mapping--step by step. Neuroimage. 2002;17(4):1665-1683.

38. Grill-Spector K, Malach R. The human visual cortex. Annu Rev Neurosci. 2004;27:649-677.

39. Duncan RO, Sample PA, Weinreb RN, Bowd C, Zangwill LM. Retinotopic organization of primary visual cortex in glaucoma: a method for comparing cortical function with damage to the optic disk. Invest Ophthalmol Vis Sci. 2007;48(2):733-744. 